\title{
Ubiquinol (reduced Coenzyme Q10) in patients with severe sepsis or septic shock: a randomized, double-blind, placebo- controlled, pilot trial
}

Michael W. Donnino ${ }^{1,2^{*}}$, Sharri J. Mortensen ${ }^{1,3}$, Lars W. Andersen ${ }^{1,4}$, Maureen Chase ${ }^{1}$, Katherine M. Berg ${ }^{2}$, Julia Balkema', Jeejabai Radhakrishnan ${ }^{5}$, Raúl J. Gazmuri ${ }^{5}$, Xiaowen Liu and Michael N. Cocchi ${ }^{1,6}$

\begin{abstract}
Introduction: We previously found decreased levels of Coenzyme Q10 (CoQ10) in patients with septic shock. The objective of the current study was to assess whether the provision of exogenous ubiquinol (the reduced form of CoQ10) could increase plasma CoQ10 levels and improve mitochondrial function.

Methods: We performed a randomized, double-blind, pilot trial at a single, tertiary care hospital. Adults (age $\geq 18$ years) with severe sepsis or septic shock between November 2012 and January 2014 were included. Patients received $200 \mathrm{mg}$ enteral ubiquinol or placebo twice a day for up to seven days. Blood draws were obtained at baseline $(0 \mathrm{~h}), 12,24,48$, and $72 \mathrm{~h}$. The primary outcome of the study was change in plasma CoQ10 parameters (total CoQ10 levels, CoQ10 levels relative to cholesterol levels, and levels of oxidized and reduced CoQ10). Secondary outcomes included assessment of: 1) vascular endothelial biomarkers, 2) inflammatory biomarkers, 3) biomarkers related to mitochondrial injury including cytochrome c levels, and 4) clinical outcomes. CoQ10 levels and biomarkers were compared between groups using repeated measures models.

Results: We enrolled 38 patients: 19 in the CoQ10 group and 19 in the placebo group. The mean patient age was $62 \pm 16$ years and $47 \%$ were female. Baseline characteristics and CoQ10 levels were similar for both groups. There was a significant increase in total CoQ10 levels, CoQ10 levels relative to cholesterol levels, and levels of oxidized and reduced CoQ10 in the ubiquinol group compared to the placebo group. We found no difference between the two groups in any of the secondary outcomes.

Conclusions: In this pilot trial we showed that plasma CoQ10 levels could be increased in patients with severe sepsis or septic shock, with the administration of oral ubiquinol. Further research is needed to address whether ubiquinol administration can result in improved clinical outcomes in this patient population.
\end{abstract}

Trial registration: Clinicaltrials.gov identifier NCT01948063. Registered on 18 February 2013.

\footnotetext{
* Correspondence: mdonnino@bidmc.harvard.edu

${ }^{1}$ Department of Emergency Medicine, Beth Israel Deaconess Medical Center,

One Deaconess Road West CC-2, Boston, MA 02215, USA

${ }^{2}$ Department of Medicine, Division of Pulmonary and Critical Care, Beth Israel

Deaconess Medical Center, One Deaconess Road West CC-2, Boston, MA, USA

Full list of author information is available at the end of the article
} 


\section{Introduction}

Severe sepsis and septic shock continue to be leading causes of morbidity and mortality despite widespread implementation of strategies focused on early antibiotic administration and aggressive resuscitation [1]. Annually there are an estimated 751,000 cases of severe sepsis in the United States, with mortality upwards of $29 \%$ [2]. Sepsis-induced multiple organ system failure is a common final pathway believed to be caused by a systemic inflammatory response involving micro- and macrocirculatory dysfunction and alterations in cellular metabolism, particularly mitochondrial dysfunction, though the exact mechanisms remain unknown [3-5]. Recent large trials of specific hemodynamic interventions have failed to show a clear benefit in patients with severe sepsis or septic shock [6-8], and new adjunct therapies are needed to improve outcomes.

Coenzyme Q10 (CoQ10) is a fat-soluble molecule synthesized in the mitochondrial inner membrane that exists both in oxidized form (ubiquinone) and reduced form (ubiquinol). The mitochondrial electron transport chain contains two transporters $(\mathrm{CoQ} 10$ and cytochrome $c)$ and four complexes (I-IV). CoQ10 plays an essential role in the electron transport chain as the carrier of electrons from complex I and II to complex III. Disruption of this mechanism can compromise oxidative phosphorylation, thereby leading to decreased levels of cellular energy (adenosine triphosphate (ATP)) production [9].

Our group has previously found decreased levels of CoQ10 in patients with septic shock [10], indicating the possibility that cellular energy production is compromised in this disease state. We also found that levels of CoQ10 were inversely associated with levels of vascular cell adhesion molecule 1 (VCAM-1) and the antiinflammatory cytokine interleukin-10 (IL-10), suggesting that CoQ10 may also play a role in vascular endothelial dysfunction and the inflammatory response seen in severe sepsis and septic shock [10].

Based on these findings, we hypothesized that CoQ10 deficiency in septic patients may contribute to mitochondrial dysfunction, and that this dysfunction could be mitigated by exogenous administration of ubiquinol (reduced CoQ10). Thus, we conducted a randomized, double-blind, placebo-controlled, pilot trial providing enteral ubiquinol supplementation to patients with severe sepsis or septic shock to determine absorption and the effect of supplementation on systemic levels, as well as the effect of ubiquinol on mitochondrial injury, markers of inflammation, and markers of vascular endothelial injury.

\section{Materials and methods}

\section{Design and setting}

This was a single-center, randomized, double-blind, pilot phase II trial comparing ubiquinol to placebo in patients with severe sepsis or septic shock. The study was conducted at Beth Israel Deaconess Medical Center (BIDMC), which is an urban tertiary care hospital in Boston, Massachusetts with approximately 55,000 emergency department visits annually and a total of 77 intensive care unit beds. The study was approved by the Committee on Clinical Investigations at BIDMC (approval number: 2012P-000267) and patients or their legally authorized surrogate provided written informed consent prior to enrollment. The trial was registered at Clinicaltrials.gov (identifier: NCT01948063) and was sponsored by Kaneka Corporation, Japan. The trial was investigator-initiated and the sponsor was not involved in study design or conduct, and had no role in manuscript preparation.

\section{Study population}

The hospital's emergency department and intensive care units were screened for eligible patients between November 2012 and January 2014. Inclusion criteria were that patients were aged $\geq 18$ years and were diagnosed with severe sepsis or septic shock. Severe sepsis was defined as the presence of two or more systemic inflammatory response syndrome criteria, documented or suspected infection, and evidence of sepsis-induced organ dysfunction or tissue hypoperfusion as defined by the Surviving Sepsis campaign [1]. Septic shock was defined as sepsis-induced hypotension persisting despite adequate fluid resuscitation, in accordance with the Surviving Sepsis campaign definitions [1].

We excluded patients based on the following criteria: 1) currently on CoQ10 supplementation, 2) unable to receive enteral medication per the clinical team, 3) enrolled in another ongoing study, 4) non-English speaking, 5) patient unable to consent and legally authorized surrogate not present, 6) 'do not resuscitate,'do not intubate' or 'comfort measures only' designation, or 7) member of a protected population.

\section{Randomization, study drug, and blinding}

A master randomization list was created using SAS (SAS Institute, Cary, NC, USA), randomizing patients to ubiquinol or placebo in a 1:1 ratio. The list was located in the central pharmacy for the duration of the study. The study drug (placebo or ubiquinol) was administered as a pill by mouth or in liquid form per nasogastric tube if present. Given the distinct taste of the liquid, this was not provided to all patients. The study drug was administered twice per day after enrollment, and was continued daily for seven days or until hospital discharge, whichever came first. Ubiquinol (Kaneka Corporation, Japan) was administered at a dosage of $200 \mathrm{mg}$ per dose. Ubiquinol was chosen over ubiquinone (oxidized CoQ10) due to its higher bioavailability [11]. The dose 
was chosen based on safety data showing that doses of $300 \mathrm{mg}$ are well-tolerated in healthy volunteers [11], and the dose was comparable to that used in a previous post-cardiac arrest trial [12]. The placebo was in identical pills or liquids as the study drug and patients, healthcare personnel, and the research team remained blinded throughout the study period.

\section{Blood samples and data collection}

Venous blood was collected at enrollment immediately before administration of study drug $(0 \mathrm{~h})$ and at 12,24 , 48 , and $72 \mathrm{~h}$ thereafter. Samples were centrifuged and serum and plasma were aliquoted into light-protected cryotubes and immediately frozen at $-80{ }^{\circ} \mathrm{C}$.

Upon enrollment, we recorded demographic data, comorbid conditions, vital signs, clinical laboratory values, whether or not the patient was receiving mechanical ventilation or vasopressors, and calculated the Acute Physiology and Chronic Health Evaluation II (APACHE II) score. Vital signs and laboratory values were also collected at all subsequent time points and outcome data, including length of intensive care unit stay, length of hospital stay, and mortality, was collected at patient discharge. All data was entered into a secure, online database by trained research assistants.

\section{Outcome measures}

The primary outcome of the study was change in plasma CoQ10 parameters (total CoQ10 levels, CoQ10 levels relative to cholesterol levels, levels of oxidized (ubiquinone) and reduced (ubiquinol) CoQ10, and the fraction of reduced CoQ10 (reduced CoQ10/total CoQ10 levels)).

Secondary outcomes included were: 1) assessment of vascular endothelial function via vascular endothelial growth factor (VEGF) and VCAM-1 measurements, 2) assessment of inflammation via IL-2, IL-6, IL-10, and tumor necrosis factor (TNF)- $\alpha$ measurements, 3 ) assessment of mitochondrial injury via cytochrome $c$ levels and DNA markers, and 4) assessment of clinical outcomes (length of ICU and hospital stay, and in-hospital mortality).

\section{Measurement of Coenzyme Q10 levels and biomarkers} CoQ10 levels were measured in plasma samples by the Department of Pathology and Laboratory Medicine at Cincinnati Children's Hospital Medical Center (Cincinnati, Ohio, USA) using high-performance liquid chromatography as previously described in detail [13]. We measured three different CoQ10 parameters (total CoQ10, oxidized CoQ10, and reduced CoQ10 (all measured in $\mu \mathrm{g} / \mathrm{mL})$ ), as well as total cholesterol levels. From these, two additional parameters were calculated: total CoQ10/cholesterol and reduced CoQ10/total
CoQ10. CoQ10 levels were measured at the 0,12 , and $24 \mathrm{~h}$ time points.

Plasma samples were analyzed for multiple vascular endothelial and inflammatory markers (VEGF, VCAM-1, IL-2, IL-6, IL-10, and TNF- $\alpha$ ) using customized Meso Scale Discovery Human Multiplex Panel (Rockville, Maryland, USA). All samples were measured in duplicate with the inter-assay coefficients of variability ranging from 2.2 to $5.8 \%$. VCAM-1 is reported in log-transformed $\mu \mathrm{g} / \mathrm{mL}$ and the rest of the markers in log-transformed $\mathrm{pg} / \mathrm{mL}$. Cytokine levels were measured at the $0,12,24,48$ and $72 \mathrm{~h}$ time points.

Cytochrome $c$ levels were measured by electrochemiluminescence in a Quickplex SQ 120 instrument (Meso Scale Discovery) at the Resuscitation Institute/ Rosalind Franklin University of Medicine and Science (North Chicago, IL, USA). Cytochrome $c$ levels had previously been reported to correlate inversely with survival after cardiac arrest in rodents [14]. Standard curves were prepared using commercially available human heart cytochrome $c$ (cat\# C3483-10UG, Sigma-Aldrich, St. Louis, MO, USA) diluted in $10 \%$ pooled human plasma from healthy volunteers in assay buffer at concentrations of $1,200.0 \mathrm{ng} / \mathrm{mL}, 400.0 \mathrm{ng} / \mathrm{mL}, 133.3 \mathrm{ng} / \mathrm{mL}, 44.4 \mathrm{ng} / \mathrm{mL}$, $14.8 \mathrm{ng} / \mathrm{mL}, 4.9 \mathrm{ng} / \mathrm{mL}, 1.6 \mathrm{ng} / \mathrm{mL}$, and $0 \mathrm{ng} / \mathrm{mL}$. Each plasma sample with an unknown cytochrome $c$ concentration was thawed in ice and diluted 1:1 in the assay buffer. Samples and standards (in a volume of $25 \mu \mathrm{L}$ ) were run in duplicate. Data analysis was performed using Meso Scale Discovery software, which developed the standard curve used to calculate the unknown concentrations. The upper limit of detection was $1,200 \mathrm{ng} / \mathrm{mL}$ and the lower limit of detection was $4 \mathrm{ng} / \mathrm{mL}$, which is defined as 2.5 standard deviations above the background mean. The software also calculates the coefficient of variation between the replicate samples. If the sample cytochrome $c$ concentration fell above or below the dynamic range of the standard curve, the assay was repeated after appropriate dilution. Cytochrome $c$ levels were expressed in log-transformed $\mathrm{ng} / \mathrm{mL}$. Cytochrome $c$ levels were measured at the 0,12 , and $24 \mathrm{~h}$ time points. Cytochrome $c$ was included as an exploratory outcome as a potential marker of mitochondrial injury [14].

We measured cell-free DNA, two markers of nuclear DNA, and two markers of mitochondrial DNA (mtDNA) at the 0,12 , and $24 \mathrm{~h}$ time points. In order to measure cell-free DNA in plasma, the plasma samples were centrifuged at $16,000 \mathrm{~g}$ for $10 \mathrm{~min}$ to remove any residual cells. The upper portion of the plasma was removed into a nuclease-free tube and stored at $-80{ }^{\circ} \mathrm{C}$ prior to DNA extraction. Cell-free DNA in the plasma was isolated using a plasma/serum DNA isolation kit (Abcam, Cambridge, MA, USA) according to the manufacturer's protocol. The amount of cell-free DNA was measured 
using a NanoDrop 1000 Spectrophotometer (Thermo Fisher Scientific, Wilmington, DE, USA). The quality of DNA was checked by the A260/280 ratio. The amount of mtDNA was measured with $m t D N A-t R N A^{\text {leu }}$ and $m t D N A-D$ loop genes, which are specifically present in the mitochondrial genome. Nuclear DNA was amplified using the single-copy beta-2-microglobulin $(B 2 M)$ and RNase $P$ nuclear genes. Primers and probes for realtime PCR were purchased from Life Technologies Corporation and utilized as previously described by Bai and Wong [15]. TaqMan real-time quantitative PCR assay was performed on a 7500 real time PCR system following the manufacturer's protocol (Applied Biosystems, Life Technologies Corporation, CA, USA). DNA extracted from whole blood with a two-fold serial dilution was used to construct the calibration curve. All samples were measured in duplicate with the inter-assay coefficients of variability ranging from 1.6 to $5.5 \%$. Cell-free DNA was measured as a marker of cellular injury, as well as a surrogate for poor outcome [16-18].

\section{Statistical analyses}

Descriptive statistics were used to summarize the study population. Data for continuous variables are presented as means with standard deviations (SD) or medians with quartiles, depending on the normality of the data. Categorical data are presented as counts with frequencies. Depending on the distribution of the data, t-tests or Wilcoxon rank sum tests were used to compare continuous data between the groups. Categorical data were compared using chi-squared or Fisher's exact tests as appropriate.

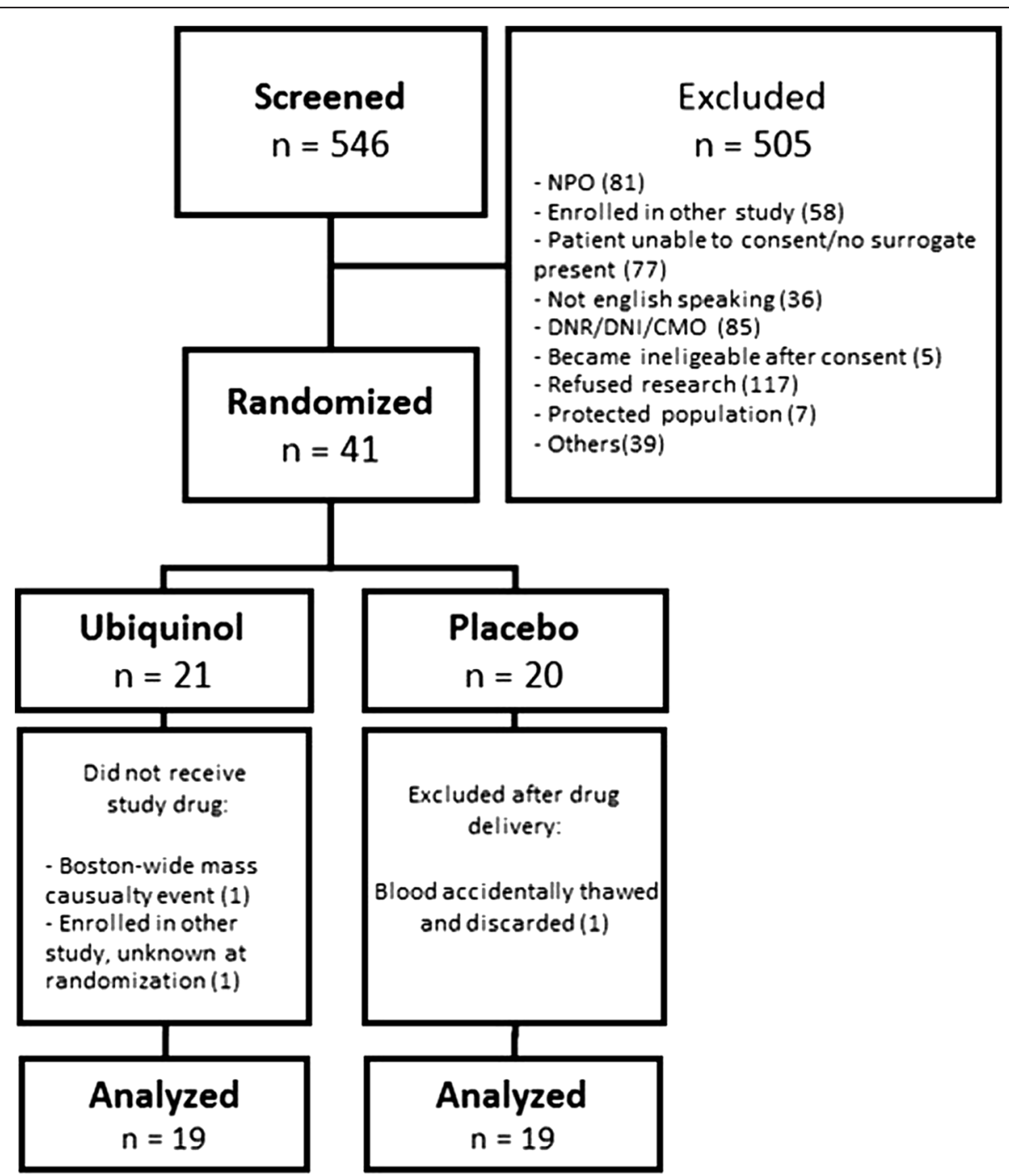

Fig. 1 Consolidated Standards for Reporting Trials (CONSORT) diagram. A total of 546 patients were screened. Of these 41 were randomized and 38 were analyzed. CMO: Comfort measures only, DNI: Do Not Intubate, DNR: Do Not Resuscitate NPO: nil per os 
Table 1 Selected baseline characteristics ${ }^{a}$

\begin{tabular}{|c|c|c|}
\hline Characteristic & Ubiquinol $(n=19)$ & Placebo $(n=19)$ \\
\hline \multicolumn{3}{|l|}{ Demographics } \\
\hline Age (years) & $60 \pm 18$ & $64 \pm 14$ \\
\hline Sex (female) & $11(58 \%)$ & $7(37 \%)$ \\
\hline Race (white) & $14(78 \%)$ & $15(83 \%)$ \\
\hline \multicolumn{3}{|l|}{ Co-morbidities } \\
\hline Coronary artery disease & $2(11 \%)$ & $2(11 \%)$ \\
\hline Congestive heart failure & $2(11 \%)$ & $2(11 \%)$ \\
\hline Hyperlipidemia & $6(32 \%)$ & $2(11 \%)$ \\
\hline Hypertension & $12(63 \%)$ & $9(47 \%)$ \\
\hline Chronic obstructive pulmonary disease & $2(11 \%)$ & $3(16 \%)$ \\
\hline Diabetes & $6(32 \%)$ & $8(42 \%)$ \\
\hline Liver disease & $1(6 \%)$ & $0(0)$ \\
\hline Renal disease & $3(16 \%)$ & $1(6 \%)$ \\
\hline Cancer & $4(21 \%)$ & $9(47 \%)$ \\
\hline \multicolumn{3}{|l|}{ Vital signs at enrollment } \\
\hline Heart rate & $92 \pm 19$ & $88 \pm 22$ \\
\hline Systolic blood pressure & $109 \pm 19$ & $105 \pm 11$ \\
\hline Diastolic blood pressure & $54 \pm 16$ & $61 \pm 11$ \\
\hline Respiratory rate & $25 \pm 6$ & $22 \pm 6$ \\
\hline Temperature & $98.4 \pm 1.4$ & $98.7 \pm 1.1$ \\
\hline \multicolumn{3}{|l|}{ Laboratory values at enrollment } \\
\hline Lactate $(\mathrm{mmol} / \mathrm{L})$ & $1.6(1.1-2.5)$ & $1.3(1.0-1.6)$ \\
\hline Creatinine (mg/dL) & $1.7(1.0-3.0)$ & $1.0(0.7-1.7)$ \\
\hline Bicarbonate (mEq/L) & $30 \pm 7$ & $34 \pm 7$ \\
\hline White blood count $(\mathrm{K} / \mu \mathrm{L})$ & $13.2 \pm 6.3$ & $16.5 \pm 11.4$ \\
\hline Hematocrit (\%) & $30.0 \pm 7.0$ & $33.7 \pm 7.4$ \\
\hline \multicolumn{3}{|l|}{ Source of sepsis } \\
\hline Pneumonia & $9(47)$ & $7(37)$ \\
\hline Urinary tract & $2(11)$ & $2(11)$ \\
\hline Intraabdominal & $1(5)$ & $3(16)$ \\
\hline Skin, joint, or soft tissue & $2(11)$ & $4(21)$ \\
\hline Endocarditis & $1(5)$ & $0(0)$ \\
\hline Unclear & $4(21)$ & $3(16)$ \\
\hline Positive blood culture & $5(26)$ & $6(32)$ \\
\hline Mechanical Ventilation & $11(58 \%)$ & $7(37 \%)$ \\
\hline Vasopressors & $12(63 \%)$ & $12(63 \%)$ \\
\hline APACHE II score at enrollment & $19 \pm 9$ & $18 \pm 10$ \\
\hline \multicolumn{3}{|l|}{ Baseline CoQ10 Levels } \\
\hline Total CoQ10 (mcg/mL) & $0.37(0.30-0.62)$ & $0.33(0.25-0.69)$ \\
\hline CoQ10 Reduced (mcg/mL) & $0.31(0.20-0.40)$ & $0.30(0.22-0.66)$ \\
\hline CoQ10 Oxidized (mcg/mL) & $0.05(0.02-0.15)$ & $0.03(0.02-0.04)$ \\
\hline CoQ10 \%Reduced & $0.87(0.73-0.94)$ & $0.92(0.90-0.95)$ \\
\hline CoQ10: Cholesterol & $0.38(0.28-0.65)$ & $0.36(0.26-0.55)$ \\
\hline
\end{tabular}

${ }^{a}$ Baseline values with continuous values expressed as means \pm standard deviation or median (quartiles), depending on normality of the data. Categorical values are counts with frequencies. No comparisons had a $P$ value $<0.05$ 
To assess the difference in repeated continuous variables (CoQ10 levels, vascular and inflammatory biomarkers, cytochrome $c$ levels, and DNA markers) between groups we used repeated measures models to account for withinsubject correlation. Variables were log-transformed before statistical analysis. An appropriate covariance structure was chosen and the residuals were visually assessed for normality. Due to the exploratory nature of the current study we did not account for multiple comparisons. Statistical analyses were conducted with the use of SAS software, version 9.3 (SAS Institute Inc., Cary, NC, USA). All hypothesis tests were two-sided, with a significance level of $P<0.05$.

\section{Results}

Study population

A total of 546 patients with severe sepsis or septic shock were screened and 41 patients were randomized. Of those, three were excluded post-randomization (two in the ubiquinol group and one in the placebo group), leaving 38 for the final analysis (see Fig. 1). The median age was $62 \pm 16$ years and $47 \%$ were female. The overall mortality was $16 \%$. There were no statistically significant differences between the baseline characteristics for the ubiquinol group and the placebo group, as illustrated in Table 1.

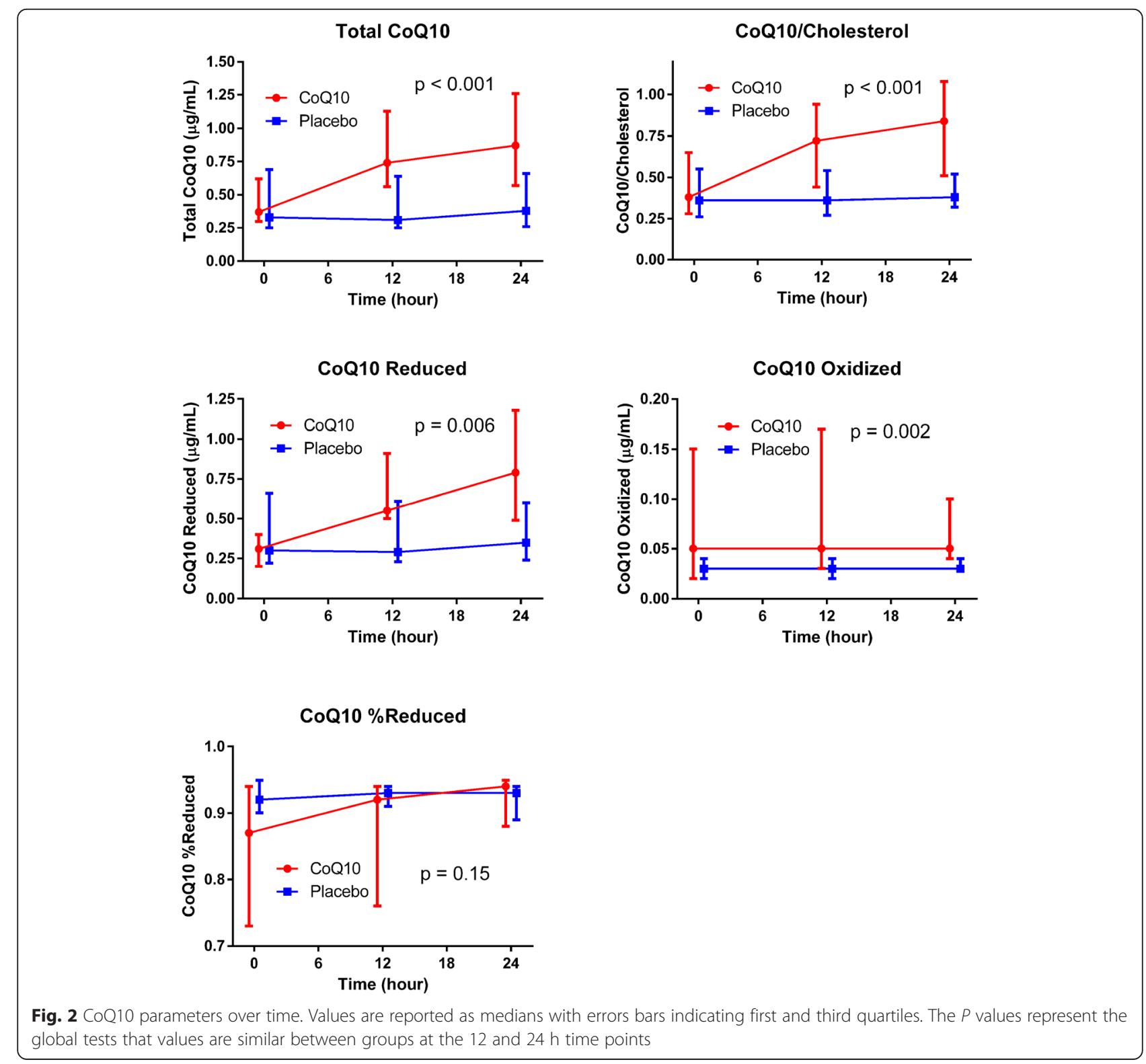




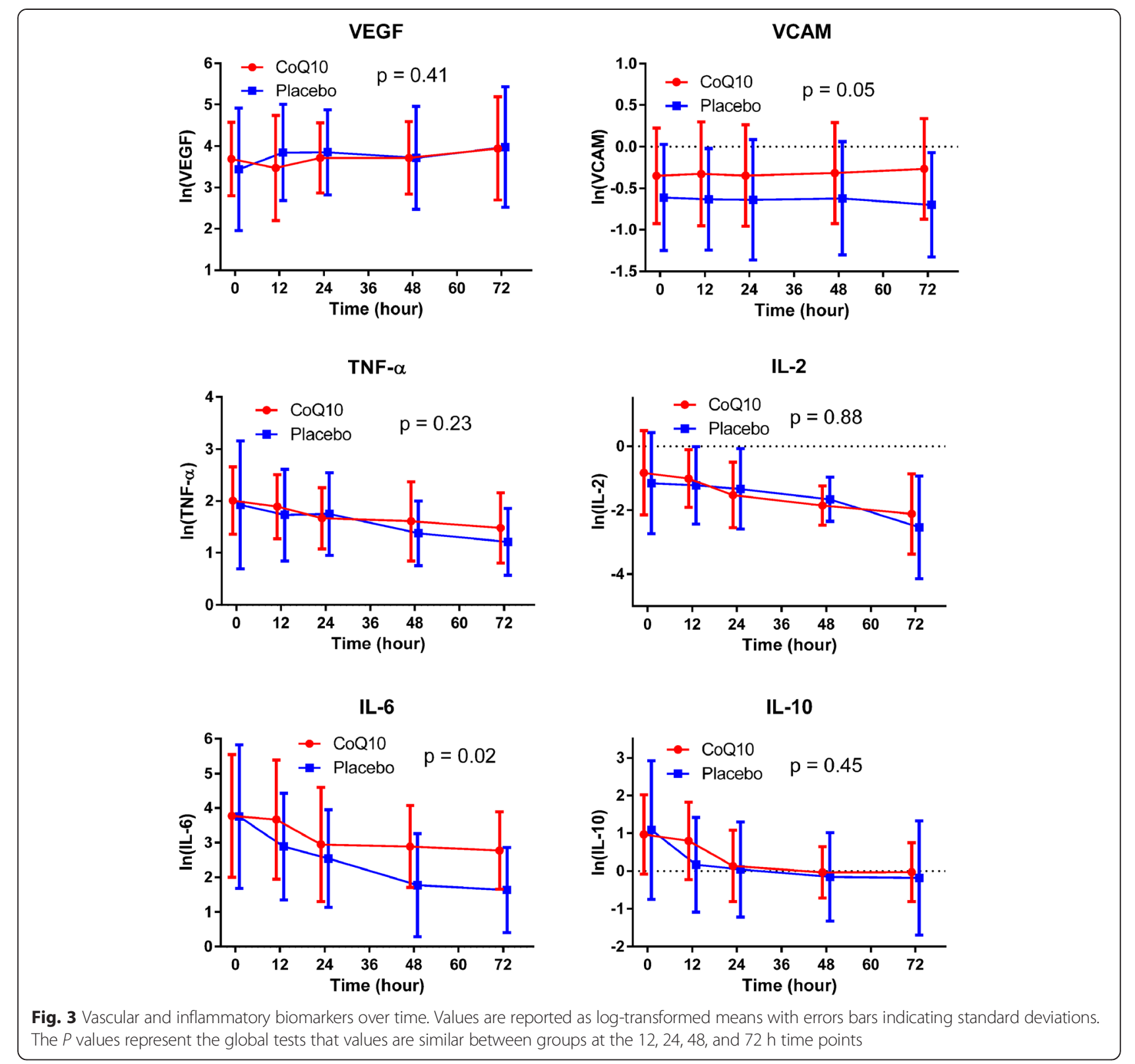

\section{Coenzyme Q10 levels}

The baseline CoQ10 levels in the two groups were similar. At 12 and $24 \mathrm{~h}$ after study drug administration there were increased levels of total CoQ10 $(P<0.001)$, CoQ10 levels relative to cholesterol levels $(P<0.001)$, reduced CoQ10 levels $(P=0.006)$ and oxidized CoQ10 levels $(P=0.002)$ in the ubiquinol-treated group compared to the placebo group. There was no statistically significant difference in the fraction of reduced CoQ10 $(P=0.15)$ (Fig. 2).

\section{Vascular endothelial and inflammatory markers}

There was no difference in baseline vascular endothelial and inflammatory markers between the groups.
IL-6 levels at $12,24,48$, and $72 \mathrm{~h}$ were higher in patients receiving ubiquinol as compared to those receiving placebo $(P=0.02)$. There was no difference between the ubiquinol and placebo groups in levels of VEGF $(P=0.41), \quad$ VCAM-1 $\quad(P=0.05), \quad$ TNF- $\alpha$ $(P=0.23), \quad$ IL-2 $\quad(P=0.88)$, or IL-10 $(P=0.45)$ over time (Fig. 3).

\section{Cytochrome c}

There was no difference in baseline cytochrome $c$ levels between groups. At 12 and $24 \mathrm{~h}$ after study drug administration, there was also no difference in cytochrome $c$ levels between groups $(P=0.32$, Fig. 4$)$. 


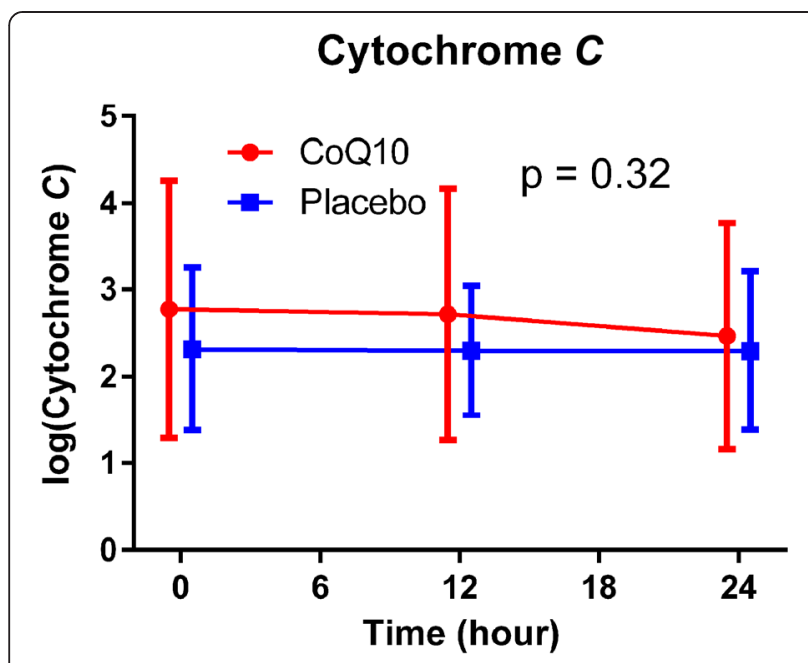

Fig. 4 Cytochrome c values over time. Values are reported as log-transformed means with errors bars indicating standard deviations. The $P$ values represent the global tests that values are similar between groups at the 12 and $24 \mathrm{~h}$ time points

\section{Nuclear and mitochondrial DNA}

There was no difference in baseline cell-free DNA, $B 2 M$, RNase $P$, mtDNA-D loop, or $m t D N A-R N A^{\text {leu }}$ between the groups. There was no difference in cell-free DNA between the two groups $(P=0.95)$. There was no difference in the fold change of $B 2 M(P=0.76)$, RNase $P(P=0.87), m t D N A-D$ loop $(P=0.58)$, or $m t D N A$ $R N A^{\text {leu }}(P=0.71)$ over time (Fig. 5).

\section{Clinical outcomes}

There was no difference in ICU length of stay (5 (3 to 7) versus 3 (2 to 6 ) days, $P=0.30$ ) or hospital length of stay (11 (6 to 19 ) days versus 10 (6 to 19 ) days, $P=0.82$ ) between the two groups. These results were similar when only analyzing survivors. There was no difference in in-hospital mortality between patients receiving ubiquinol versus placebo (4 (21\%) versus $2(11 \%), P=0.66)$. The four deaths in the ubiquinol group happened 1, 1, 5, and 16 days after the drug administration. The mode of death was determined to be comorbid withdrawal of care in two patients with underlying terminal illness, refractory hypotension in one patient with underlying terminal illness, and progressive respiratory failure in one patient with underlying alcohol-associated hepatic failure. The mode of death for the two patients in the placebo group was determined as comorbid withdrawal of care in one patient with terminal illness, and refractory hypotension in one patient with underlying terminal illness (both deaths occurred four days after drug administration). As noted, the majority of deaths occurred in patients with a preexisting terminal illness, including metastatic cancer or end-stage organ failure. None of the deaths were determined to be related to the study medication as assessed by an independent data safety monitoring board. Gastrointestinal disturbance, potentially related to ubiquinol administration, was reported in one patient.

\section{Discussion}

In this pilot study of ubiquinol versus placebo in patients with severe sepsis and septic shock, plasma CoQ10 levels increased in patients who received ubiquinol. We found statistically significant higher levels of IL-6 in patients receiving ubiquinol as compared to those receiving placebo. The reason for this finding remains largely unexplained, but could be related to multiple testing. The assessment of other biomarkers related to vascular endothelial function, mitochondrial dysfunction, and inflammation revealed no statistically significant difference between the ubiquinol and the placebo groups in this study.

CoQ10, as a key component of the electron transport chain, plays an important role in the mitigation of oxidative stress and production of cellular energy [19]. Previous investigations have demonstrated that CoQ10 levels are low in critical illness, specifically septic shock and post-cardiac arrest [10, 20, 21]. Multiple rat models have been used to test and demonstrate the beneficial impact of CoQ10 in sepsis and have found decreased oxidative stress, prevention of mitochondrial damage, less renal and acute liver dysfunction, and neuroprotective effects [22-24]. In the current study, we demonstrate that exogenous provision of ubiquinol increases CoQ10 levels in patients with severe sepsis and septic shock. The potential benefits of this increase remain unclear.

Severe sepsis is associated with respiratory, cardiovascular, and renal dysfunction, with significant alterations to metabolic pathways and cellular function [25, 26]. A central hypothesis in organ dysfunction is the concept of tissue hypoxia [27]. Cytopathic hypoxia (compromised cellular oxygen utilization), rather than inadequate oxygen delivery, may play a significant role in the development of multi-organ dysfunction and lactate elevation in sepsis $[28,29]$, although the exact mechanisms remain unknown [30]. Despite optimization of hemodynamics and oxygen therapy, oxygen may not be utilized at the mitochondrial level. Mitochondria consume $90 \%$ of cellular oxygen in order to generate energy as ATP, via oxidative phosphorylation by transfer of electrons from the Krebs cycle to the electron transport chain, via nicotinamide adenine dinucleotide and 5,10-methylenetetrahydrofolate reductase [31]. The electron transport chain is located on the inner mitochondrial membrane and consists of four complexes and two transporters. CoQ10 plays an essential role in the electron transport chain as the carrier of electrons from complex I and II to complex III. Disruption of this mechanism can compromise oxidative phosphorylation in the mitochondria, 
Cell-free DNA

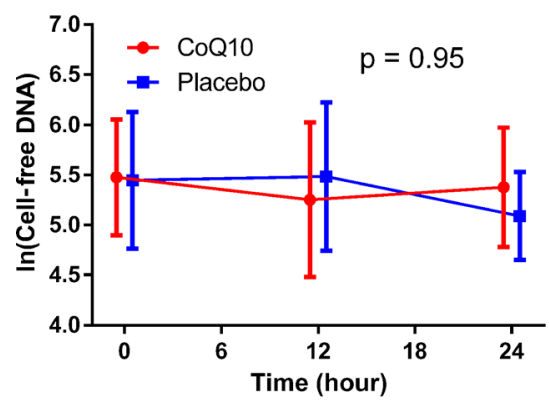

Beta-2-microglobulin

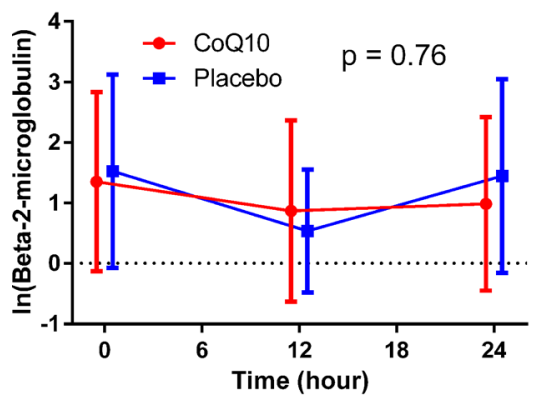

mtDNA-D loop

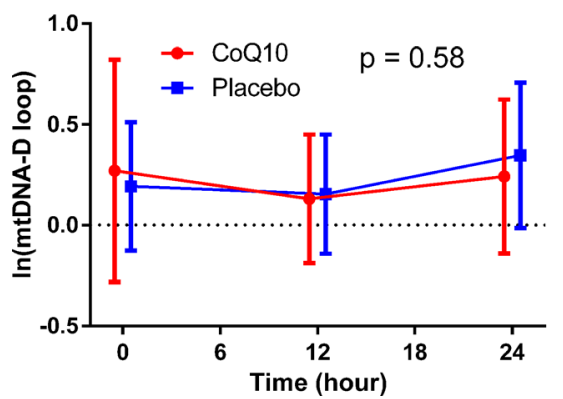

RNase P

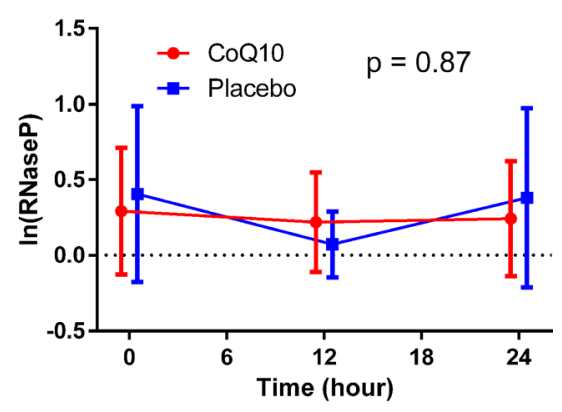

mtDNA-RNA ${ }^{\text {leu }}$

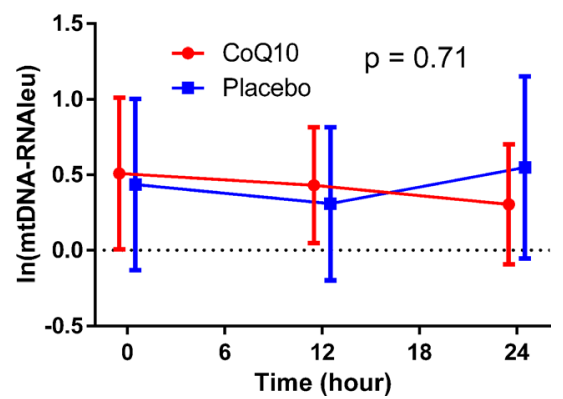

Fig. 5 Cell-free DNA, and nucleus and mitochondrial DNA values over time. Values are reported as log-transformed means with errors bars indicating standard deviations. The $P$ values represent the global tests with values that are similar between groups at the 12 and $24 \mathrm{~h}$ time points

thereby leading to decreased levels of cellular ATP production [9].

Previous studies have demonstrated a beneficial role of CoQ10 as a co-adjutant in the treatment of syndromes characterized by impaired mitochondrial bioenergetics and increased oxidative stress [32]. CoQ10 has shown beneficial effects on diastolic and systolic function in patients with chronic heart failure [33-35] and, in a single-center pilot trial, CoQ10 was found to increase three-month survival when given to post-cardiac arrest patients [12]. Moreover CoQ10 has shown promising clinical benefits in small human studies of progressive supranuclear palsy [36], Huntington's disease [37], and Friedreich's ataxia [38]. Finally, CoQ10 has also been studied in Parkinson's disease, with inconsistent results [39-41].
In the current study we were unable to detect any differences in secondary endpoints between the groups. There are multiple possible explanations for this lack of effect. Our sample size was relatively small, and we may have been underpowered to detect clinically relevant differences in biomarkers or clinical outcomes. A relatively large portion of potentially eligible patients were excluded, which may decrease the generalizability of our findings. Given the lack of human trials in septic patients, we used a relatively low dose $(200 \mathrm{mg}$ twice per day). Previous studies have demonstrated that oral consumption of CoQ10 at dosages of 400,600, 800, and $1,200 \mathrm{mg}$ per day are well tolerated in patients with Parkinson's disease, with the greatest benefit in the group receiving the highest dosage $(1,200 \mathrm{mg}$ per day) $[40,39]$. As such, our dose may have been inadequate to 
show a treatment effect, and higher doses of ubiquinol may be required to compensate for the severity of metabolic dysfunction and energy depletion that occurs in severe sepsis and septic shock.

The timing and duration of our intervention may have influenced the results. Approximately half of our patients were mechanically ventilated and comatose at the time of enrollment, which led to some delay in the administration of the first dose as consent had to be obtained from a legally authorized surrogate. The duration of therapy was relatively short (maximum of seven days), and a longer duration and follow-up period may be needed, especially for long-term patient-centered outcomes. For example, ubiquinol could theoretically protect against cognitive dysfunction or muscular weakness post-septic shock, but we did not measure these longerterm outcomes. Further, the majority of our biomarker endpoints were only assessed within the first $24 \mathrm{~h}$. It is possible that changes could have been seen if the endpoints were evaluated at later time points. The enrolled patient population was heterogeneous, with a relatively low overall mortality (16\%) that, based on review by a blinded physician, was mainly related to withdrawal of care in patients with underlying terminal illness. Future studies might consider excluding patients with terminal illness. Whether certain subgroups, such as a more acutely ill population with potential signs of mitochondrial injury (for example, elevated lactate), may benefit remains unknown. Lastly, the possibility remains that ubiquinol is ineffective as a 'metabolic resuscitator' in patients with severe sepsis and septic shock. However, additional studies are needed before any firm conclusions can be made.

\section{Conclusions}

We have shown that ubiquinol is absorbed in patients with severe sepsis and septic shock. There were no significant changes in VEGF, VCAM-1, the majority of inflammatory markers, or clinical outcomes, such as mortality and length of ICU and hospital stay. Further investigation with different dosages, timing of drug delivery, or more specific populations may be warranted to determine whether ubiquinol administration may be beneficial as an adjunct therapy in patients with severe sepsis and septic shock.

\section{Key messages}

- Coenzyme Q10 is an essential component of the mitochondrial electron transport chain.

- In this placebo-controlled, randomized, pilot trial we found that enteral administration of ubiquinol (reduced coenzyme Q10) increases total Coenzyme Q10 levels, Coenzyme Q10 levels relative to cholesterol levels, and levels of oxidized and reduced Coenzyme Q10.

- There was no difference in vascular endothelial biomarkers, inflammatory biomarkers, or biomarkers related to mitochondrial injury, including cytochrome c levels.

- We found no difference in clinical outcomes between the ubiquinol and placebo groups.

\section{Abbreviations}

APACHE II: Acute Physiology and Chronic Health Evaluation II; ATP: Adenosine triphosphate; B2M: Beta-2-microglobulin; BIDMC: Beth Israel Deaconess Medical Center; CoQ10: Coenzyme Q10; ICU: Intensive care unit; IL: Interleukin; mtDNA: Mitochondrial DNA; SD: Standard deviations; TNF: Tumor necrosis factor; VCAM-1: Vascular cell adhesion molecule; VEGF: Vascular endothelial growth factor.

\section{Competing interests}

Kaneka Corporation, Japan sponsored this trail. The investigation was investigator-initiated and the sponsor was not involved in study design or conduct, and had no role in manuscript preparation. The authors declare that they have no competing interests.

\section{Authors' contributions}

MWD and MNC were responsible for study concept and design. MWD, MC $\mathrm{KMB}$, and $\mathrm{MNC}$ were responsible for coordination of the trial. SJM and JB were responsible for acquisition of data. JR, RJG, and XL performed biomarker analysis. MWD, SJM, and LWA drafted the manuscript. LWA conducted the statistical analysis. All authors provided intellectual input and interpretation of the data, critically revised the manuscript for important intellectual content, and approved the final version for submission. All authors agree to be accountable for all aspects of the work and have read and approved the final manuscript.

\section{Acknowledgements}

Dr. Donnino is supported by the NHLBI (1K02HL107447.01A1. Dr. Berg is supported by the American Heart Association (13CRP16930000). Dr. Cocchi is supported by the American Heart Association (14SDG22420010). The authors wish to thank Francesca Montillo for her editorial support in submitting the manuscript. The authors wish to acknowledge and thank the members of the Data Safety Monitoring Board, Dr. Peter Clardy, Beth Israel Deaconess Medical Center and Dr. David Leaf, Brigham and Women's Hospital.

\section{Author details}

'Department of Emergency Medicine, Beth Israel Deaconess Medical Center, One Deaconess Road West CC-2, Boston, MA 02215, USA. ${ }^{2}$ Department of Medicine, Division of Pulmonary and Critical Care, Beth Israel Deaconess Medical Center, One Deaconess Road West CC-2, Boston, MA, USA. ${ }^{3}$ Research Center for Emergency Medicine, Aarhus University Hospital, Norrebrogade 44, Aarhus 8000, Denmark. ${ }^{4}$ Department of Anesthesiology, Aarhus University Hospital, Norrebrogade 44, Aarhus 8000, Denmark. ${ }^{5}$ Resuscitation Institute at Rosalind Franklin University of Medicine and Science, 3333 Green Bay Road, North Chicago, IL 60064, USA. ${ }^{6}$ Department of Anesthesia Critical Care, Division of Critical Care, Beth Israel Deaconess Medical Center, One Deaconess Road West CC-2, Boston, MA, USA.

Received: 19 February 2015 Accepted: 15 June 2015

Published online: 01 July 2015

\section{References}

1. Dellinger RP, Levy MM, Rhodes A, Annane D, Gerlach H, Opal SM, et al. Surviving sepsis campaign: international guidelines for management of severe sepsis and septic shock: 2012. Crit Care Med. 2013;41:580-637. doi:10.1097/CCM.0b013e31827e83af.

2. Angus DC, Linde-Zwirble WT, Lidicker J, Clermont G, Carcillo J, Pinsky MR. Epidemiology of severe sepsis in the United States: analysis of incidence, outcome, and associated costs of care. Crit Care Med. 2001;29:1303-10. 
3. Sakr Y, Dubois MJ, De Backer D, Creteur J, Vincent JL. Persistent microcirculatory alterations are associated with organ failure and death in patients with septic shock. Crit Care Med. 2004;32:1825-31.

4. Harrois A, Huet O, Duranteau J. Alterations of mitochondrial function in sepsis and critical illness. Curr Opin Anaesthesiol. 2009;22:143-9. doi:10.1097/ACO.0b013e328328d1cc.

5. De Backer D, Orbegozo Cortes D, Donadello K, Vincent JL. Pathophysiology of microcirculatory dysfunction and the pathogenesis of septic shock. Virulence. 2014;5:73-9. doi:10.4161/viru.26482.

6. Investigators PCESS, Yealy DM, Kellum JA, Huang DT, Barnato AE, Weissfeld $L A$, et al. A randomized trial of protocol-based care for early septic shock. N Engl J Med. 2014;370:1683-93. doi:10.1056/NEJMoa1401602.

7. Asfar P, Meziani F, Hamel JF, Grelon F, Megarbane B, Anguel N, et al. High versus low blood-pressure target in patients with septic shock. N Engl J Med. 2014;370:1583-93. doi:10.1056/NEJMoa1312173.

8. Arise Investigators and the Anzics Clinical Trials Group, Peake SL, Delaney A, Bailey M, Bellomo R, Cameron PA, et al. Goal-directed resuscitation for patients with early septic shock. N Engl J Med. 2014;371:1496-506. doi:10.1056/NEJMoa1404380.

9. Singer $M$. The role of mitochondrial dysfunction in sepsis-induced multi-organ failure. Virulence. 2014;5:66-72. doi:10.4161/viru.26907.

10. Donnino MW, Cocchi MN, Salciccioli JD, Kim D, Naini AB, Buettner C, et al. Coenzyme Q10 levels are low and may be associated with the inflammatory cascade in septic shock. Crit Care. 2011;15:R189. doi:10.1186/cc10343.

11. Hosoe K, Kitano M, Kishida H, Kubo H, Fujii K, Kitahara M. Study on safety and bioavailability of ubiquinol (Kaneka QH) after single and 4-week multiple oral administration to healthy volunteers. Regul Toxicol Pharmacol. 2007:47:19-28. doi:10.1016/j.yrtph.2006.07.001.

12. Damian MS, Ellenberg D, Gildemeister R, Lauermann J, Simonis G, Sauter W, et al. Coenzyme Q10 combined with mild hypothermia after cardiac arrest: a preliminary study. Circulation. 2004;110:3011-6. doi:10.1161/01.CIR.0000146894.45533.C2

13. Tang PH, Miles MV, DeGrauw A, Hershey A, Pesce A. HPLC analysis of reduced and oxidized coenzyme Q(10) in human plasma. Clin Chem. 2001:47:256-65.

14. Radhakrishnan J, Wang S, Ayoub IM, Kolarova JD, Levine RF, Gazmuri RJ. Circulating levels of cytochrome $\mathrm{c}$ after resuscitation from cardiac arrest: a marker of mitochondrial injury and predictor of survival. Am J Physiol Heart Circ Physiol. 2007;292:H767-75. doi:10.1152/ajpheart.00468.2006.

15. Bai RK, Wong L. Simultaneous detection and quantification of mitochondrial DNA deletions, depletion, and over-replication in patients with mitochondrial disease. J Mol Diagn. 2005;7:613-22 doi:10.1016/S1525-1578(10)60595-8.

16. Saukkonen K, Lakkisto P, Pettila V, Varpula M, Karlsson S, Ruokonen E, et al. Cell-free plasma DNA as a predictor of outcome in severe sepsis and septic shock. Clin Chem. 2008:54:1000-7. doi:10.1373/clinchem.2007.101030.

17. Dwivedi DJ, Toltl LJ, Swystun LL, Pogue J, Liaw KL, Weitz Jl, et al. Prognostic utility and characterization of cell-free DNA in patients with severe sepsis. Crit Care. 2012;16:R151. doi:10.1186/cc11466.

18. Avriel A, Paryente Wiessman M, Almog Y, Perl Y, Novack V, Galante O, et al. Admission cell free DNA levels predict 28-day mortality in patients with severe sepsis in intensive care. PLoS One. 2014:9:e100514. doi:10.1371/journal.pone.0100514.

19. Turunen $\mathrm{M}$, Olsson J, Dallner G. Metabolism and function of coenzyme Q. Biochim Biophys Acta. 2004;1660:171-99.

20. Cocchi MN, Giberson B, Berg K, Salciccioli JD, Naini A, Buettner C, et al. Coenzyme Q10 levels are low and associated with increased mortality in post-cardiac arrest patients. Resuscitation. 2012;83:991-5. doi:10.1016/j.resuscitation.2012.03.023.

21. Coppadoro A, Berra L, Kumar A, Pinciroli R, Yamada M, Schmidt UH, et al. Critical illness is associated with decreased plasma levels of coenzyme Q10: a cross-sectional study. J Crit Care. 2013;28:571-6. doi:10.1016/j.jcrc.2013.02.009.

22. Chuang YC, Chan JY, Chang AY, Sikorska M, Borowy-Borowski H, Liou CW, et al. Neuroprotective effects of coenzyme Q10 at rostral ventrolateral medulla against fatality during experimental endotoxemia in the rat. Shock. 2003;19:427-32. doi:10.1097/01.shk.0000048900.46342.37.

23. Lowes DA, Thottakam BM, Webster NR, Murphy MP, Galley HF. The mitochondria-targeted antioxidant MitoQ protects against organ damage in a lipopolysaccharide-peptidoglycan model of sepsis. Free Radic Biol Med 2008:45:1559-65. doi:10.1016/j.freeradbiomed.2008.09.003.

24. Supinski GS, Murphy MP, Callahan LA. MitoQ administration prevents endotoxin-induced cardiac dysfunction. Am J Physiol Regul Integr Comp Physiol. 2009;297:R1095-102. doi:10.1152/ajpregu.90902.2008.

25. American College of Chest Physicians/Society of Critical Care Medicine Consensus Conference: definitions for sepsis and organ failure and guidelines for the use of innovative therapies in sepsis. Crit Care Med. 1992;20:864-74.

26. Trager K, DeBacker D, Radermacher P. Metabolic alterations in sepsis and vasoactive drug-related metabolic effects. Curr Opin Crit Care. 2003;9:271-8.

27. Crouser ED. Mitochondrial dysfunction in septic shock and multiple organ dysfunction syndrome. Mitochondrion. 2004;4:729-41. doi:10.1016/j.mito.2004.07.023

28. Fink MP. Cytopathic hypoxia. Mitochondrial dysfunction as mechanism contributing to organ dysfunction in sepsis. Crit Care Clin. 2001;17:219-37.

29. Garcia-Alvarez M, Marik P, Bellomo R. Sepsis-associated hyperlactatemia. Crit Care. 2014;18:503-14. doi:10.1186/s13054-014-0503-3.

30. Andersen LW, Mackenhauer J, Roberts JC, Berg KM, Cocchi MN, Donnino MW. Etiology and therapeutic approach to elevated lactate levels. Mayo Clin Proc. 2013;88:1127-40. doi:10.1016/j.mayocp.2013.06.012.

31. McBride HM, Neuspiel M, Wasiak S. Mitochondria: more than just a powerhouse. Curr Biol. 2006;16:R551-60. doi:10.1016/j.cub.2006.06.054.

32. Littarru GP, Tiano L. Clinical aspects of coenzyme Q10: an update. Nutrition. 2010;26:250-4. doi:10.1016/j.nut.2009.08.008.

33. Kocharian A, Shabanian R, Rafiei-Khorgami M, Kiani A, Heidari-Bateni G. Coenzyme Q10 improves diastolic function in children with idiopathic dilated cardiomyopathy. Cardiol Young. 2009;19:501-6. doi:10.1017/S1047951109990795.

34. Adarsh K, Kaur H, Mohan V. Coenzyme Q10 (CoQ10) in isolated diastolic heart failure in hypertrophic cardiomyopathy (HCM). Biofactors. 2008;32:145-9.

35. Sander S, Coleman Cl, Patel AA, Kluger J, White CM. The impact of coenzyme Q10 on systolic function in patients with chronic heart failure. J Card Fail. 2006;12:464-72. doi:10.1016/j.cardfail.2006.03.007.

36. Stamelou M, Reuss A, Pilatus U, Magerkurth J, Niklowitz P, Eggert KM, et al. Short-term effects of coenzyme Q10 in progressive supranuclear palsy: a randomized, placebo-controlled trial. Mov Disord. 2008;23:942-9. doi:10.1002/mds.22023.

37. Koroshetz WJ, Jenkins BG, Rosen BR, Beal MF. Energy metabolism defects in Huntington's disease and effects of coenzyme Q10. Ann Neurol. 1997:41:160-5. doi:10.1002/ana.410410206.

38. Cooper JM, Korlipara LV, Hart PE, Bradley JL, Schapira AH. Coenzyme Q10 and vitamin E deficiency in Friedreich's ataxia: predictor of efficacy of vitamin E and coenzyme Q10 therapy. Eur J Neurol. 2008;15:1371-9. doi:10.1111/j.1468-1331.2008.02318.x

39. Shults CW, Oakes D, Kieburtz K, Beal MF, Haas R, Plumb S, et al. Effects of coenzyme Q10 in early Parkinson disease: evidence of slowing of the functional decline. Arch Neurol. 2002;59:1541-50.

40. Shults CW, Beal MF, Fontaine D, Nakano K, Haas RH. Absorption, tolerability, and effects on mitochondrial activity of oral coenzyme Q10 in parkinsonian patients. Neurology. 1998;50:793-5.

41. Parkinson Study Group QEl, Beal MF, Oakes D, Shoulson I, Henchcliffe C, Galpern WR, et al. A randomized clinical trial of high-dosage coenzyme Q10 in early Parkinson disease: no evidence of benefit. JAMA Neurol. 2014;71:543-52. doi:10.1001/jamaneurol.2014.131

\section{Submit your next manuscript to BioMed Central and take full advantage of:}

- Convenient online submission

- Thorough peer review

- No space constraints or color figure charges

- Immediate publication on acceptance

- Inclusion in PubMed, CAS, Scopus and Google Scholar

- Research which is freely available for redistribution 